\title{
Alimentação natural de Etropus crossotus Jordan \& Gilbert (Teleostei, Pleuronectiformes, Paralichthyidae), na Armação do Itapocoroy, Penha, Santa Catarina, Brasil
}

\author{
Maria José Lunardon-Branco \& Joaquim Olinto Branco
}

Centro de Ciências Tecnológicas da Terra e do Mar, Universidade do Vale do Itajaí. Caixa Postal 360, 88301-970 Itajaí, Santa Catarina, Brasil.E-mail: mbranco@cttmar.univali.br

\begin{abstract}
Natural feeding of Etropus crossotus Jordan \& Gilbert, 1882 in the Armação do Itapocoroy, Santa Catarina, Brazil. The feeding of $E$. crossotus was analyzed during the period from January to December 1995 using the frequency of occurrence, frequency of points and feeding index methods. The species presents a broad trophic specter and diversified, composed by 32 itens. The trophic analyses indicated the species show that the categories Crustacea, Polychaeta and Osteichthyes were the most exploited resources.
\end{abstract}

KEY WORDS. Artisanal fishery, feeding habits, seasonal changes.

Etropus crossotus Jordan \& Gilbert, 1882 (Linguado) pertence à família Paralichthyidae que distribui-se no Atlântico desde Chesapeake Bay (USA), Golfo do México, América Central, incluindo Trinidad, Venezuela, até o litoral Sul do Brasil; freqüentemente ocorrem em águas pouco profundas em fundos de areia, com conchas; predominam em substratos biodetríticos (Topp \& Hoff 1972; Figueiredo \& Menezes 2000).

O estudo da alimentação natural de organismos aquáticos, evoluiu com o tempo, sendo desenvolvidas metodologias que atuam como ferramentas para auxiliar na avaliação e compreensão da disponibilidade dos itens presas consumidos. $\mathrm{O}$ método mais adequado a ser empregado para peixes, deve ser o que permita melhor compreender o espectro alimentar das espécies (BERG 1979). Existem poucas informações disponíveis sobre a bioecologia dos linguados no litoral brasileiro, especialmente enfocando os hábitos alimentares das espécies. Assim, este trabalho tem como objetivo contribuir para o conhecimento do hábito alimentar de Etropus crossotus, através da análise do conteúdo estomacal.

\section{MATERIAL E MÉTODOS}

As coletas foram realizadas mensalmente, durante o período de janeiro a dezembro de 1995, em área tradicionalmente utilizada pelos pescadores artesanais do camarão sete-barbas (Xiphopenaeus kroyeri Heller, 1862) entre 6,0 a 10,0 metros de profundidade na Armação do Itapocoroy, Penha, Santa Catarina $\left(26^{\circ} 46^{\prime}-26^{\circ} 47^{\prime} \mathrm{S}\right.$ e $48^{\circ} 36^{\prime}-48^{\circ} 37^{\prime} \mathrm{W}$ ) (BRANCO et al. 1998). Na captura dos camarões e da sua fauna acompanhante foram utilizadas duas redes-de-arrasto com portas, com 6,0 m de aber- tura de boca; malhas de 3,0 cm na manga e no corpo e 2,0 cm no ensacador; o tempo de cada arrasto foi de 30 minutos com velocidade média da baleeira de dois nós.

Os linguados coletados foram acondicionados em sacos plásticos e mantidos em caixa de isopor com gelo, a fim de retardar o processo de digestão. Após a coleta, os exemplares foram mantidos em "freezer" até serem processados. Os estômagos foram retirados com auxílio de tesoura e pinça; inicialmente pesados, abertos e estimado o grau de repleção estomacal determinado de acordo com a quantidade de alimento presente nos estômagos. Em seguida, o conteúdo estomacal foi removido com jatos de água (frasco lavador) e depositado em placa de Petri com água.

A identificação dos itens alimentares foi realizada com auxílio de bibliografia especializada. Os itens do conteúdo que não puderam ser identificados, devido ao elevado grau de digestão, foram considerados como "Matéria Orgânica Não Determinada" (MOND). Neste estudo, foi assumido que não ocorrem diferenças significativas entre o volume relativo de alimento consumido por machos e fêmeas de E. crossotus, sendo a dieta analisada para sexos agrupados.

Para a análise quali-quantitativa foram aplicados os métodos dos Pontos (MP) e da Freqüência de Ocorrência (FO) (Hynes 1950, Hyslop 1980, Berg 1979), complementados pelo índice alimentar modificado de KaWAKAmI \& VAzzoler (1980), o que contribuiu para a compreensão global da dieta de $E$. crossotus, tornando possíveis comparações com outras espécies e as inter-relações predador-presa. A diversidade e a equitabilidade foram calculados e aplicados às categorias alimentares para a espécie, conforme Ludwig \& ReYNolds (1988). 


\section{RESULTADOS E DISCUSSÃO}

Foram analisados 418 estômagos de E. crossotus, sendo que $45,45 \%$ desse encontravam-se vazios e $54,55 \%$ com algum conteúdo (Tab. I).

Tabela I. Distribuição de freqüência absoluta (N) e percentual (\%) de estômagos vazios e com conteúdo, durante o período de estudo para Etropus crossotus.

\begin{tabular}{lcc}
\hline Estado de repleção & $\mathrm{N}$ & $\%$ \\
\hline Vazio & 190 & 45,45 \\
Com conteúdo & 228 & 54,55 \\
\hline Total & 418 & 100,00 \\
\hline
\end{tabular}

O espectro alimentar de $E$. crossotus mostrou-se diversificado composto por 32 itens, sendo a categoria Crustacea, com o maior número de espécies exploradas (Tab. II). Dentre eles, os itens mais representativos em relação ao volume relativo (MP) ocupado nos estômagos foram Amphipoda (33,12\%), Gammaridea $(22,74 \%)$ e Cumacea $(7,41 \%)$, os demais itens deste grupo representaram volumes inferiores a $2,4 \%$. Em relação à freqüência com que esses itens ocorreram nos estômagos, os mais representativos foram Amphipoda, presentes em 27,19\% dos estômagos, seguidos por Gammaridea $(17,77 \%)$ e Cumacea $(9,42 \%)$ (Tab. II). A segunda categoria alimentar em importância foi Polychaeta que representou $18,97 \%$ do volume estomacal, estando presente em 18,20\% dos estômagos analisados. Osteichthyes, foi freqüente em 7,28\% dos estômagos, ocupando $3,29 \%$ do seu volume. Os demais itens foram menos representativos tanto em relação a sua freqüência, quanto ao volume ocupado. Etropus crossotus apresentou uma baixa porcentagem de areia, a qual não representou importância trófica, mas uma conseqüência de seu modo de captura das presas (Tab. II).

A dieta de E. crossotus, na Armação do Itapocoroy, mostrou uma semelhança com aquelas encontradas para outros Pleuronectiformes no litoral do Ceará, São Paulo, Rio Grande do Sul, Maldonado e litoral de Netherlands (Thizzssen et al. 1974, KaWAKAmi \& Amaral 1983, Almeida et al. 1997).

Ao longo das estações do ano, ocorreram variações no tipo de alimento explorado, porém, a categoria mais importante foi Peracarida (Figs 1, 2). Durante o verão, os Peracarida foram o principal recurso alimentar utilizado pelo exemplares de E. crossotus, seguido dos Polychaeta e dos demais Crustacea. As categorias Echinodermata, Osteichthyes e Cnidaria foram exploradas acidentalmente (Fig. 1a).

No outono, Peracarida foi a base da dieta sendo Crustacea geral o segundo recurso mais explorado seguido por Polychaeta. As algas, foram ingeridas apenas nesta estação e raramente juntamente com Cnidaria. O grupo Osteichthyes foi predado ocasionalmente (Fig. 1b).

No inverno e na primavera, a base da alimentação continuou sendo Peracarida e a segunda categoria mais utilizada foi Polychaeta, sendo os demais grupos ingeridos raramente (Fig. $2 \mathrm{a}, \mathrm{b})$.

De maneira geral, E. crossotus apresentou um espectro alimentar diversificado, sendo Crustacea a principal fonte de alimento. A espécie também utilizou Polychaeta como alternati-
Tabela II. Freqüência de ocorrência (\%) e de pontos (\%) dos itens alimentares durante o período de estudo para Etropus crossotus.

\begin{tabular}{|c|c|c|c|c|}
\hline Itens & MP & $\%$ & $\mathrm{FO}$ & $\%$ \\
\hline Algae & 57 & 0,39 & 2 & 0,43 \\
\hline Macrophyta & 2 & 0,01 & 1 & 0,21 \\
\hline \multicolumn{5}{|l|}{ Cnidaria } \\
\hline Renilla sp. & 32 & 0,22 & 3 & 0,64 \\
\hline Anthozoa & 30 & 0,20 & 1 & 0,21 \\
\hline Nematoda & 4 & 0,03 & 2 & 0,43 \\
\hline \multicolumn{5}{|l|}{ Mollusca } \\
\hline Gastropoda & 2 & 0,01 & 1 & 0,21 \\
\hline \multicolumn{5}{|l|}{ Annelida } \\
\hline Polychaeta & 2767 & 18,80 & 84 & 17,99 \\
\hline Nereidae & 25 & 0,17 & 1 & 0,21 \\
\hline \multicolumn{5}{|l|}{ Crustacea } \\
\hline Stomatopoda & 15 & 0,10 & 1 & 0,21 \\
\hline Decapoda & 272 & 1,85 & 8 & 1,71 \\
\hline Penaeus sp. & 227 & 1,54 & 4 & 0,86 \\
\hline Sergestidae & 15 & 0,10 & 1 & 0,21 \\
\hline Acetes americanus & 52 & 0,35 & 2 & 0,43 \\
\hline Caridea & 100 & 0,68 & 2 & 0,43 \\
\hline Alpheidae & 2 & 0,01 & 1 & 0,21 \\
\hline Hepatus pudibundus & 25 & 0,17 & 1 & 0,21 \\
\hline Portunus spinimanus & 127 & 0,86 & 3 & 0,64 \\
\hline Brachyura & 351 & 2,39 & 15 & 3,21 \\
\hline Pinnotheridae & 81 & 0,55 & 6 & 1,28 \\
\hline Grapsidae & 25 & 0,17 & 1 & 0,21 \\
\hline Mysidacea & 210 & 1,43 & 11 & 2,36 \\
\hline Cumacea & 1090 & 7,41 & 44 & 9,42 \\
\hline Gammaridea & 3346 & 22,74 & 83 & 17,77 \\
\hline Caprella sp. & 25 & 0,17 & 1 & 0,21 \\
\hline Amphipoda & 4874 & 33,12 & 127 & 27,19 \\
\hline Isopoda & 139 & 0,94 & 6 & 1,28 \\
\hline Tanaidacea & 96 & 0,65 & 7 & 1,50 \\
\hline Ovos Crustacea & 47 & 0,32 & 3 & 0,64 \\
\hline \multicolumn{5}{|l|}{ Echinodermata } \\
\hline Ophiuroidea & 52 & 0,35 & 2 & 0,43 \\
\hline Osteichthyes & 484 & 3,29 & 34 & 7,28 \\
\hline Matéria Orgânica & 111 & 0,75 & 7 & 1,50 \\
\hline Areia & 30 & 0,20 & 2 & 0,43 \\
\hline Total & 14715 & 100,00 & 467 & 100,00 \\
\hline
\end{tabular}



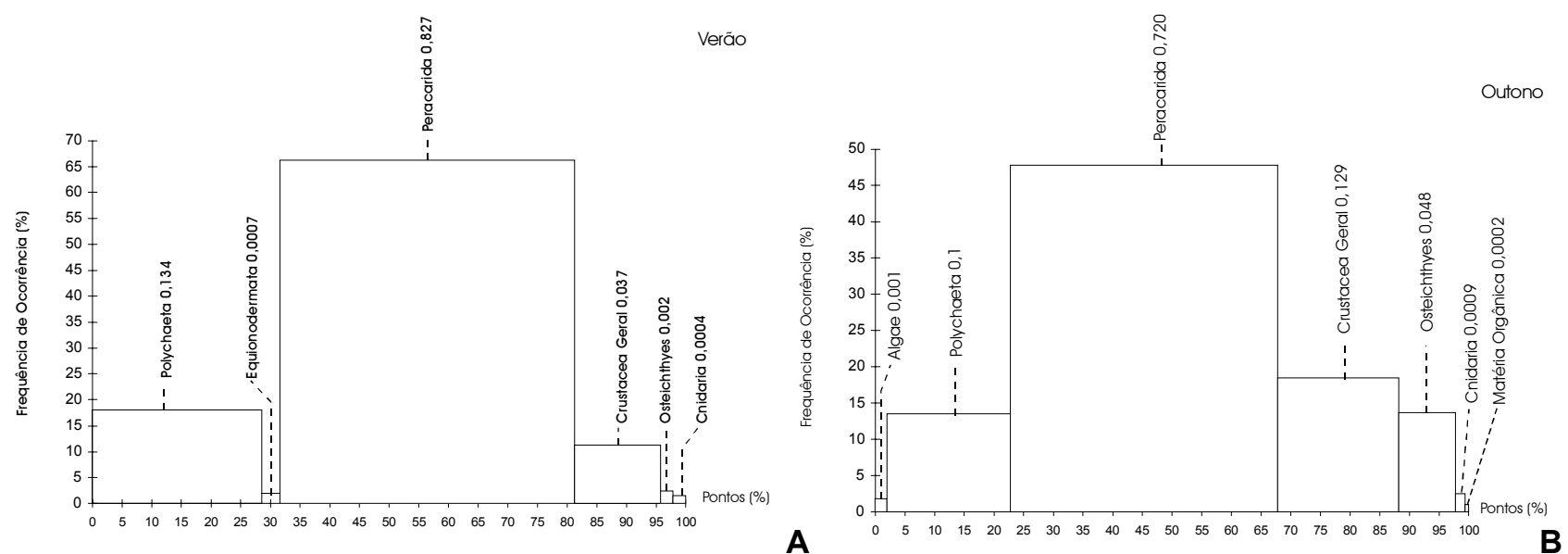

Figura 1. Freqüência de ocorrência (\%) e dos pontos (\%) dos grupos alimentares e seus respectivos índices nas estações de verão (A) e outono (B) para Etropus crossotus.
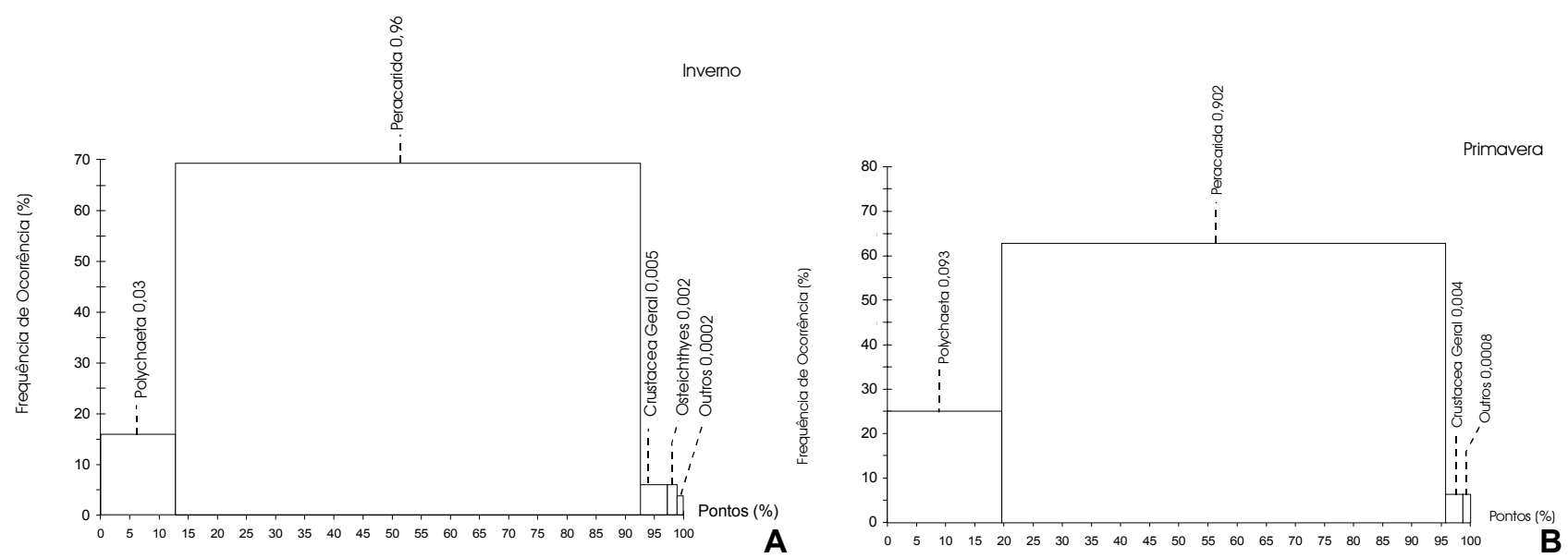

Figura 2. Freqüência de ocorrência (\%) e dos pontos (\%) dos grupos alimentares e seus respectivos índices nas estações de inverno (A) e primavera (B) para Etropus crossotus.

va alimentar, assim como Algae, Macrophyta, Echinodermata e Osteichthyes que foram explorados raramente (Fig. 3).

De acordo com SOAREs et al. (1993), a dieta de E. crossotus constou basicamente de Polychaeta, Gammaridae e larvas de Brachyura. Embora tivesse apresentado um espectro alimentar bastante diversificado, Polychaeta foi o principal grupo consumido. Neste estudo, E. crossotus, também apresentou um espectro alimentar diversificado, entretanto, a principal presa ingerida foi Crustacea, seguida de Polychaeta. Essa variação na exploração de presas pode estar associado a sua disponibilidade no ambiente, e aos movimentos migratórios da fauna.

Amaral \& Migotto (1980) e Kawakami \& Amaral (1983), ressaltaram a importância dos Polychaeta na alimentação de muitas espécies de peixes demersais e também daquelas que apresentam hábitos bentônico, como no caso de Etropus longimanus Norman, 1933 e Symphurus jenynsi Evermann \& Kendall, 1907, entre outros Pleuronectiformes da plataforma continental entre Torres-Maldonado. Esses estudos mostraram haver uma

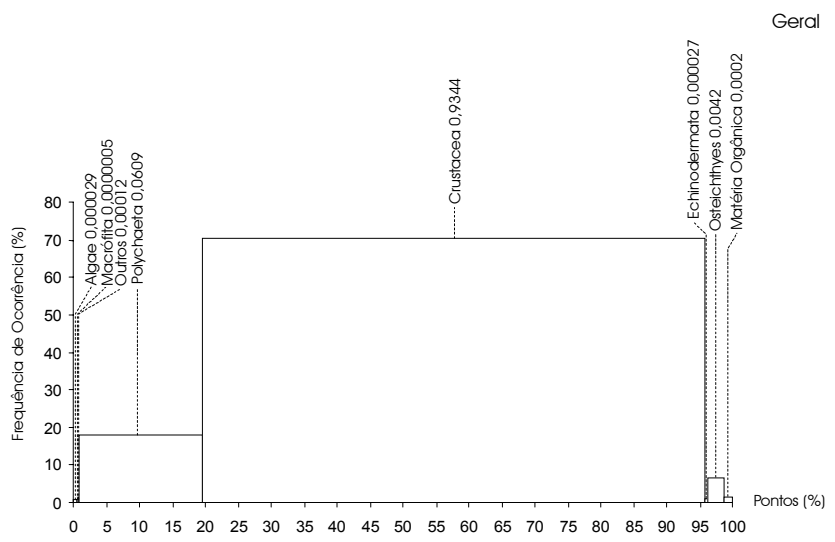

Figura 3. Freqüência de ocorrência (\%) e dos pontos (\%) dos grupos alimentares e seus respectivos índices durante o período de estudo (Outros: Cnidaria, Mollusca, Nematoda) para Etropus crossotus.

Revista Brasileira de Zoologia 20 (4): 631-635, dezembro 2003 
forte relação entre a maior ocorrência destas espécies em determinados tipos de fundo associados a presença dos Polychaeta, os quais participam de modo significativo na cadeia alimentar das populações bentônicas e demersais.

Como em outros Pleurinectiformes, os Nematoda apareceram quase que freqüentemente, embora não tivessem sido registrados sua abundância. Fitzhugh \& FleEger (1985), Coull et al. (1989) e Ellis \& Coull (1989) consideram que esse taxa apresente uma migração vertical no sedimento, principalmente no momento em que o peixe está se alimentando, deste modo, sua ingestão passa a ser acidental e mostraram que em predadores com determinado tamanho e que apresentem o comportamento de "mordiscar" o sedimento, esse grupo não representa a principal fonte de alimento.

Os índices de diversidade de Shannon-Wiener $\left(\mathrm{H}^{\prime}\right)$ e equitabilidade de Pielou $\left(J^{\prime}\right)$ foram calculados sazonalmente para as categorias alimentares (Tab. III). No verão o valor da diversidade foi de 1,0197 com a menor equitabilidade $56,91 \%$ entre as estações, indicando que a exploração dos recursos não ocorreu de maneira uniforme. Entretanto, não foram registradas ocorrências extremamente reduzidas dos diferentes grupos alimentares.

Tabela III. Variação sazonal dos índices de diversidade (H' e Hmax) e de equitabilidade (J'), aplicado às categorias alimentares de Etropus crossotus.

\begin{tabular}{lcccc}
\hline \multirow{2}{*}{ Estações } & \multirow{2}{*}{$\begin{array}{c}\text { Categorias } \\
\text { alimentares }\end{array}$} & \multicolumn{3}{c}{ Índices } \\
\cline { 3 - 5 } & & $\mathrm{H}^{\prime}$ (nats) & Hmax (nats) & $\mathrm{E}=\mathrm{J}^{\prime}(\%)$ \\
\hline Verão & 6 & 1,0197 & 1,7918 & 56,91 \\
Outono & 7 & 1,4186 & 1,9459 & 72,90 \\
Inverno & 5 & 0,9893 & 1,6094 & 61,47 \\
Primavera & 4 & 0,9583 & 1,3863 & 69,12 \\
\hline
\end{tabular}

No outono, com o aumento do número de categorias alimentares, foram registrados maiores valores de diversidade $(1,4186)$ e de equitabilidade $(72,90 \%)$ sugerindo que a utilização das categorias foi mais uniforme quando comparado com o observado no verão (Fig. 1b).

No inverno, os valores da diversidade e da equitabilidade foram baixos devido ao reduzido número de categorias utilizadas, onde houve um acentuado destaque para o grupo Peracarida. O mesmo aconteceu na Primavera resultando na menor diversidade entre as estações com uma uniformidade na exploração das presas. Assim, os recursos alimentares quando comparados com o inverno, foram explorados mais igualmente. Mesmo assim, Peracarida destacou-se entre as categorias alimentares ingeridas (Fig. 2a, b).

De acordo com Werner \& Hall (1974), Morais \& Bodiou (1984), Nelson \& Coull (1989), Gill \& Hart (1998), Lunardon (1990), LUNARDON et al. (1991), o comportamento alimentar está relacionado com o tamanho da presa, da boca do predador, com a morfologia do aparelho digestório, capacidade estomacal e com a estratégia de forrageamento. Os mecanismos empregados pelos peixes para sua alimentação, representam adaptações que os capacitam a tomada de um tipo específico de presa (Labropoulou \& Papadopoulou-Smith 1999).
Na Armação do Itapocoroy, E. crossotus mostrou uma estratégia de forrageamento eficiente, visto a gama de organismos predados, incluindo matéria vegetal. O trato digestório desta espécie apresenta esôfago e estômago relativamente grandes em relação a maioria dos peixes, intestino com volta simples e cecos pilóricos quase ausentes; esta configuração está diretamente relacionada com o tipo de presa que compõe a dieta do linguado. A habilidade desses peixes em localizar a sua presa, seleciona-la e captura-la são fatores importantes para o sucesso de sua alimentação (Labropoulou \& PAPAdopoulou-Smith 1999).

De acordo com o comportamento alimentar, E. crossotus é classificado como sendo do tipo "plaice", pois arqueia seu corpo e projeta sua boca para capturar a presa (YAZDANI 1969). Essa estratégia permite selecionar sua presa. No caso dos Polychaeta, na maioria das vezes, são encontrados apenas a cabeça ou fragmentos inteiros do corpo no estômago, isto acontece pela sua apreensão com a mandíbula guarnecida com dentículos finos (Topp \& Holf 1972).

Com base nesses resultados, pode-se considerar $E$. crossotus segundo a natureza dos alimentos ingeridos, como onívora/carcinófaga e segundo a diversidade de itens explorados como eurifágica.

Na Armação do Itapocoroy, E. crossotus utilizou como principal fonte de alimento Crustacea que incluiu tanto animais com grande mobilidade com é o caso dos Dendrobranchiata, como aqueles mais lentos (Peracarida) caracterizando uma estratégia alimentar do tipo "tubot" (YAZDANI 1969) que inclui peixes que se alimentam de presas com maior mobilidade. Esse comportamento mostra a grande plasticidade da espécie na busca e na captura das presas, uma vez que pode ser incluído nas duas modalidades.

\section{REFERÊNCIAS BIBLIOGRÁFICAS}

Almeida, Z.S.; V. FonsêCa-Genevois \& A.L. Vasconcelos-Filho. 1997. Alimentação de Achirus lineatus (Teleostei, Pleuronectiforme: Achiridae) em Itapissuma, PE. Boletim do Laboratório de Hidrobiologia, São Luis, (10): 79-95.

Amaral, A.C.Z. \& A. Migotto. 1980. Importância dos anelídeos poliquetas na alimentação da macrofauna demersal e epibentônica da região de Ubatuba. Boletim do Instituto Oceanográfico, São Paulo, 29 (2): 31-35.

BERG, J. 1979. Discussion of methods of investigating the food of fishes, with a reference to a preliminary study of the prey of Gobiusculus flavenscens (Gobiidae). Marine Biology 50: 263-273.

Branco, J.O.; M.J. Lunardon-Branco; A.C. Peret; F.X. Souto; R. Schweitzer \& W.G. Vale. 1998. Associações entre macroinvertebrados e peixes demersais na Armação do Itapocoroy, Penha, SC, Brasil. Brazilian Archives of Biology and Tecnology, Curitiba, 41(2): 268-277.

Coull, C.B.; M.A. Palmer \& P.E. Myers. 1989. Controls on the vertical distribuition of meiobenthos in mud: field and flume studies with juvenile fish. Marine Ecology Progress Series 55: 133-139.

EluIs, M.J. \& B.C. CoulL. 1989. Fish predation on meiobenthos: field experiments with juveniles spot Leiostomus xanthurus Lacépède. Journal of Experimental Marine Biology and Ecology 130: 19-32.

Figueiredo, J.L. \& N. Menezes. 2000. Manual de peixes marinhos do Sudeste do Brasil. VI. Teleostei (5). São Paulo, 
Museu de Zoologia, Universidade de São Paulo, 116p.

Fitzhugh, G.R. \& J.W. Fleeger. 1985. Goby (Pisces: Gobiidae) interactions with meiofauna and small macrofauna. Bulletin of Marine Science, Miami, 36: 436-444.

Gill, A.B. \& P.J.B. HarT. 1998. Stomach capacity as a directing factor in prey size selection of three-spined stickleback. Journal of Fish Biology 53 (4): 897-900.

Hynes, H.B.N. 1950. The food of fresh-water sticklebacks (Gasterosteus aculeatus and Pygosteus pungitius), with a review of methods used in studies of the food of fishes. Journal Animal Ecology 19 (1): 36-51.

HysLop, E.J. 1980. Stomach contents analysis - a review of methods and their application. Journal of Fish Biology 17: 411-429.

KAWAKAMI, E. \& A.C.Z. AMARAL. 1983. Importância dos anelídeos poliquetas no regime alimentar de Etropus longimanus Normann, 1908 (Pisces, Pleuronectiformes). Heringia, Série Zoologia, Porto Alegre, 62: 47-54.

KaWAKAMI, E. \& G. VAzzoler. 1980. Método gráfico e estimativa de índice alimentar aplicado no estudo de alimentação de peixes. Boletim do Instituto Oceanográfico, São Paulo, 29 (2): 205-207.

Labropoulou, M. \& K.N. Papadopoulou-Smith. 1999. Foraging behaviour patterns of four simpatric demersal fishes. Estuarine and Coastal and Shelf Science, London, 49 (Supl. A): 99-108.

LUDWIG, J.A. \& J.F. REYNOLDS. 1988. Statistical ecology: a primer on methods and computing. New York, John Wiley \& Sons, 338p.

Lunardon, M.J. 1990. Hábitos alimentares de Menticirrhus littoralis (Holbrook, 1860) (Perciformes - Sciaenidae) na Baía de Paranaguá e adjacências - Paraná - Brasil. Arquivos de Biologia e Tecnologia, Curitiba, 33 (3): 717-725.

Lunardon. M.J.; J.L.E. Silva; J.R. Verani \& J.O. Branco. 1991. Comportamento alimentar de Menticirrhus americanus (Linnaeus, 1758) (Perciformes: Sciaenidae) no litoral do Paraná, Brasil. Arquivos de Biologia e Tecnologia, Curitiba, 34 (3/4): 487-502.

Morais, L.T. \& J.Y. Bodiou. 1984. Predation on meio fauna by juvenile fish in a Western Mediterranean flatfish nursery ground. Marine Biology 82 (2): 209-215.

Nelson, A.L. \& B.C. Coull. 1989. Selection of meiobenthic prey by juvenile spot (Pisces): a experimental study. Marine Ecology Progress Series, Curitiba, 53: 51-57.

Soares, L.S.H.; M.A. Gasalla; M.A.T. Rios; M.V. Arrasa \& C.L.Del B. Rossi-Wongtschowski. 1993. Grupos tróficos de onze espécies dominantes de peixes demersais da plataforma continental interna de Ubatuba, Brasil. Publicação Especial do Instituto Oceanográfico, São Paulo, (10): 189-198.

Thijssen, R.; A.J. Lever \& J. Lever. 1974. Food composition and feeding periodicity of O-Group plaice (Pleuronectes platessa) in the tidal area of a sandy beach. Netherlands Journal Sea Research 8 (4): 369-377.

Topp, R.W. \& F.H.J.R. Hoff. 1972. Flatfishes (Pleuronectiformes). Mém. Hourglass Cruises. Fla. Dept. Resources Mar. Res. Lab 4 (Part 2): 135.

Werner, E.E. \& J.D. Hall. 1974. Optimal foraging and size selection of prey by the bluegill sunfish (Lepomis macrochirus), Ecology 55 (5): 1042-1052.

YAZDANI, G.M. 1969. Adaptation in the jaws of flatfish (Pleuronectiformes). Journal of Zoology 159: 181-222.

Recebido em 11.VI.2003; aceito em 20.X.2003. 